\title{
COMPARISON OF THE EFFICIENCY OF CAFFEINE VERSUS AMINOPHYLLINE FOR THE TREATMENT OF APNOEA OF PREMATURITY
}

\author{
Gabriela Ildiko Zonda ${ }^{1}$, Andreea Avasiloaiei ${ }^{1}$, Mihaela Moscalu ${ }^{2}$, Maria Stamatin ${ }^{\mathbf{1}}$ \\ ${ }^{1}$ Department of Mother and Child Medicine, Regional Neonatal Intensive Care Unit, \\ "Cuza-Voda" Hospital of Obstetrics and Gynaecology, \\ "Gr. T. Popa" University of Medicine and Pharmacy, Iasi \\ ${ }^{2}$ Department of Preventive Medicine and Interdisciplinarity, \\ "Gr. T. Popa" University of Medicine and Pharmacy, Iasi
}

\begin{abstract}
Purpose. Comparison of the efficiency of caffeine and aminophylline for the treatment of apnoea in premature neonates.

Material and method. We conducted a retrospective study on two lots of premature infants admitted in the Regional Centre of Neonatal Intensive Care Unit of the "Cuza-Vodă" Clinical Hospital of Obstetrics and Gynaecology of lasi during June 2012 - June 2015.

Results. Neonates treated with caffeine had significantly less apnoea on day 3 and 14 of treatment comparing with those who received aminophylline. Caffeine treatment was associated with less need for CPAP and mechanical ventilation comparing with aminophylline, but there was no statistical significance. Anemia, intraventricular hemorrhage, persistent ductus arteriosus and chronic lung disease were correlated with an increased duration of the treatment for apnoea of prematurity. Neonates from the caffeine group had more episodes of agitation, tachycardia and digestive intolerance, but at the same time a reduced incidence of necrotizing enterocolitis, weight loss and hypertension, compared to those from the aminophylline group. The hospital stay duration was reduced in the infants that received caffeine treatment compared to those that received aminophylline. Conclusion. The therapeutic advantages of caffeine are remarkable due to low incidence of significant adverse effects and its long-term cost/efficiency ratio, which recommend it as the best treatment for apnoea of prematurity. These facts justify the initiation of a multicentre national study including a higher number of premature neonates for the evaluation of the efficiency of caffeine therapy in all gestational age groups.
\end{abstract}

Keywords: apnoea, prematurity, methylxanthines, caffeine

\section{INTRODUCTION}

Apnoea of prematurity, defined as the cessation of breathing for at least 20 seconds, or for less than 20 seconds, but associated with bradycardia or cyanosis, in a neonate with a gestational age below 37 weeks (1), is a consequence of the physiologic immaturity of the centres that control breathing. The incidence and severity of apnoea increase as the gestational age decreases. Apnoeic episodes in preterm infants may have central cause (absent inspiratory efforts), obstructive mechanism (airway obstruction with present inspiratory efforts), or may be mixed (combination of central and obstructive mechanisms), with latter being the most common form (2).

Severe apnoeic episodes with duration of more than 20 seconds are often accompanied by bradycardia and desaturation which can lead to disturbances in cerebral haemodynamic and neurodevelopmental impairment (3). Treatment for apnoea of prematurity includes prone positioning, methylxanthines and CPAP (continuous positive airway pressure) or NIPPV (nasal intermittent positive pressure ventilation). Doxapram is a potent respiratory stimulant which increases minute-volume by increasing the respiratory rate in adult patients. Its use has been documented in newborns with recur-

Corresponding author:

Andreea Avasiloaiei, "Gr. T. Popa" University of Medicine and Pharmacy, 16 Universitatii Street, lasi

E-mail: andreea.avasiloaiei@umfiasi.ro 
rent apnoea despite methylxanthine treatment, but its routine use is controversial because of the potential side effects and the lack of long term data (4).

Other therapeutic measures include sensory stimulation, $\mathrm{CO}_{2}$ inhalation and packed red cells transfusion, but they require additional studies and are not recommended as routine treatment (5).

Recurrent apnoea represents a frequent pathology in premature infants, the incidence and severity being inversely correlated with the gestational age (6). Apnoea can be a spontaneous event attributed solely to prematurity, but it can also be triggered or aggravated by factors like infections, hypoxemia, anaemia, metabolic disturbances, intracranial pathology, gastroesophageal reflux or certain drugs. Due to the well-known stimulatory effect of the methylxanthines on the respiratory centre, they have been used for treatment of apnoea since the 1970's. Their efficiency compared to placebo has been evaluated in a Cochrane review which has established their role in reducing apnoeic events, but the mechanism of action could not be specified. Possibilities include increased chemoreceptor responsiveness (based on increased breathing responses to $\mathrm{CO}_{2}$ ), enhanced respiratory muscle performance and generalized central nervous system excitation (7).

Caffeine has a potential therapeutic advantage over aminophylline due to its higher therapeutic ratio, more reliable enteral absorption and longer half-life, which allows once daily administration (8).

The purpose of this paper is to compare the efficiency of two methylxanthines, caffeine and aminophylline, for the treatment of apnoea in premature infants.

\section{MATERIAL AND METHOD}

We conducted a retrospective study on two groups of premature neonates admitted in the Regional Centre of Neonatal Intensive Care Unit of the "Cuza-Vodă" Clinical Hospital of Obstetrics and Gynaecology of Iasi during June 2012 - June 2015. The first group included 46 premature infants admitted in our unit between June $1^{\text {st }}$ and December $31^{\text {st }} 2012$, who received aminophylline as a loading dose of $5 \mathrm{mg} / \mathrm{kg}$ at extubation, followed by a maintenance dose of $2-3 \mathrm{mg} / \mathrm{kg}$ divided in 2 daily doses until the apnoea resolved. The second group included 301 premature neonates admitted between January $1^{\text {st }} 2013$ and June $30^{\text {th }} 2015$ who received caffeine as a loading dose of $20 \mathrm{mg} / \mathrm{kg}$ followed by a maintenance dose of $5 \mathrm{mg} / \mathrm{kg}$ in a single daily dose.
In both groups we analysed the following parameters: gestational age (GA), birth weight (BW), Apgar score at 1, 5 and 10 minutes, primary pathology, the need and duration of mechanical ventilation, the moment when the methylxanthine treatment was initiated, the duration of administration, the number, severity and evolution of apnoeic spells during treatment, associated pathology which could have influenced the duration and severity of apnoea (anaemia, patent ductus arteriosus, chronic lung disease) and the duration of hospital stay. Chronic lung disease was defined as need for supplemental oxygen at 28 days or 36 weeks postconceptional age (9).

\section{RESULTS}

Of the total preterm neonates with a $\mathrm{GA} \leq 35$ weeks admitted between June 2012 and June 2015 in our unit, $22.61 \%$ had apnoea. The incidence varies inversely with the gestational age: $61.38 \%(\leq 29$ wks.), $43.03 \%$ (30-31 wks.), 18.8\% (32-33 wks.), $2.99 \%$ (34-35 wks.), respectively.

In the first group (aminophylline), the mean GA was 29.13 wks. ( $\min =25$ wks., $\max =34$ wks.), and the mean birth weight was $1174.3 \mathrm{~g}(\mathrm{~min}=650 \mathrm{~g}$, $\max =1,900 \mathrm{~g}$ ). In the second group (caffeine), the mean GA was 29.92 wks. $(\min =25$ wks., $\max =35$ wks. $)(p=0.037534 ; C I=95 \%)$, and the mean birth weight was $1325.1 \mathrm{~g}(\min =500 \mathrm{~g}, \max =2,560 \mathrm{~g})$ ( $p=0.007798 ; C I=95 \%)$. The median value for the Apgar score at 1, 5 and 10 minutes respectively was 5, 7 and 7 for group 1, and 6, 7 and 7 for group $2\left(\mathrm{p}_{1}=0.187460, \mathrm{p}_{5}=0.100796, \mathrm{p}_{10}=0.109985 ; \mathrm{CI}\right.$ $=95 \%)$.

In both groups the primary pathology was represented mainly by respiratory distress syndrome due to surfactant deficiency $(89.13 \%$ vs. $60.13 \%$; $\mathrm{p}=0.00014 ; \quad \mathrm{CI}=95 \%)$, followed by congenital pneumonia, (4.35\% vs. $9.30 \%$; $\mathrm{p}=0.265$; $\mathrm{CI}=95 \%)$, pulmonary haemorrhage $(2.17 \%$ vs. $2.33 \%$; $\mathrm{p}=0.948 ; \mathrm{CI}=95 \%)$ and pneumothorax $(2.17 \% \mathrm{vs}$ $2.66 \% ; \mathrm{p}=0.847 ; \mathrm{CI}=95 \%$ ).

The analysis of the data concerning the need for mechanical ventilation as treatment for primary pathology, showed that $17.39 \%$ of the infants from group 1 (aminophylline) and $11.63 \%$ from group 2 (caffeine) were ventilated with HFOV (high frequency oscillatory ventilation $)(\mathrm{p}=0.26919 ; \mathrm{CI}=$ $95 \%), 73.91 \%$ of the neonates from group 1 , and $58.8 \%$ from group 2 with IPPV (intermitent positive pressure ventilation) $(\mathrm{p}=0.04532 ; \mathrm{CI}=95 \%)$, and $50 \%$ of the neonates from group 1 , and $36.5 \%$ from group 2 were ventilated with SIMV (synchro- 
nized intermittent mechanical ventilation) $(\mathrm{p}=0.08044 ; \mathrm{CI}=95 \%) .95 .65 \%$ of the patients from group 1, and $84.96 \%$ of the patients from group 2 needed non-invasive respiratory support like CPAP ( $\mathrm{p}=0.02849$; $\mathrm{CI}=95 \%)$.

In both groups we analysed the duration of invasive $(\mathrm{HFOV}+\mathrm{IPPV}+\mathrm{SIMV})$ or non-invasive respiratory support (CPAP), and the data showed a shorter duration in infants treated with caffeine comparing with those who received aminophylline,

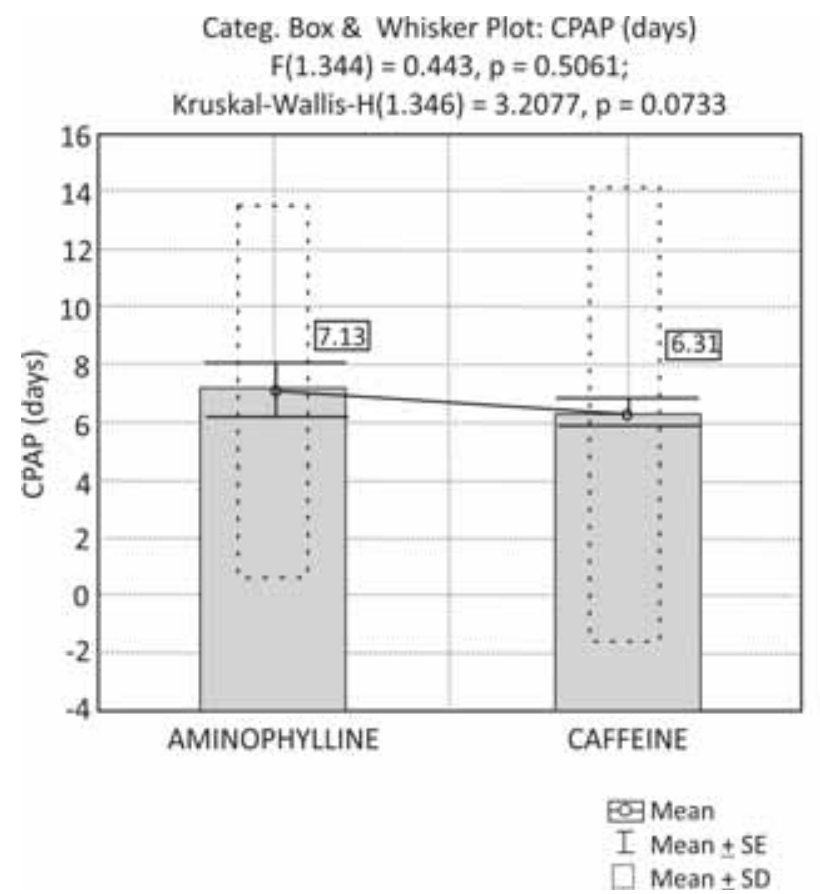

Categ, Box \& Whisker Plot: Mechanical ventilation (days) $F(1.345)=2.6515, p=0.1044$; $\mathrm{KW}-\mathrm{H}(1.347)=8.1904, \mathrm{p}=0.0042$

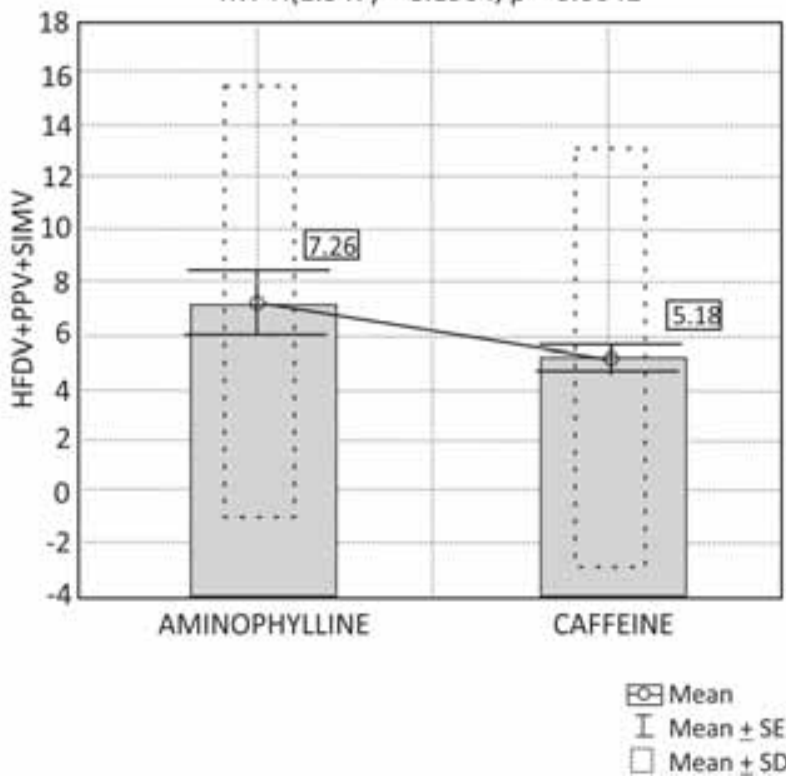

FIGURE 1. Statistical indicators for the duration of respiratory support as treatment for the primary pathology $C P A P$ - continuous positive airway pressure
5.18 vs. 7.26 days $(p=0.104364 ; \mathrm{CI}=95 \%)$ for mechanical ventilation and 6.31 vs. 7.13 days $(\mathrm{p}=0.506142 ; \mathrm{CI}=95 \%)$ for CPAP, respectively (Fig. 1). Although the differences in the mean duration of ventilation are significant for both invasive support and CPAP, the correlation cannot be considered statistically relevant because of the major size inequality between the two study groups.

The analysis of apnoea frequency after the initiation of methylxanthine treatment revealed a statistically relevant reduction of apnoeic events after day 3 and $14^{\text {th }}$ of treatment in the neonates from the caffeine group, compared to those from the aminophylline group (Fig. 2).

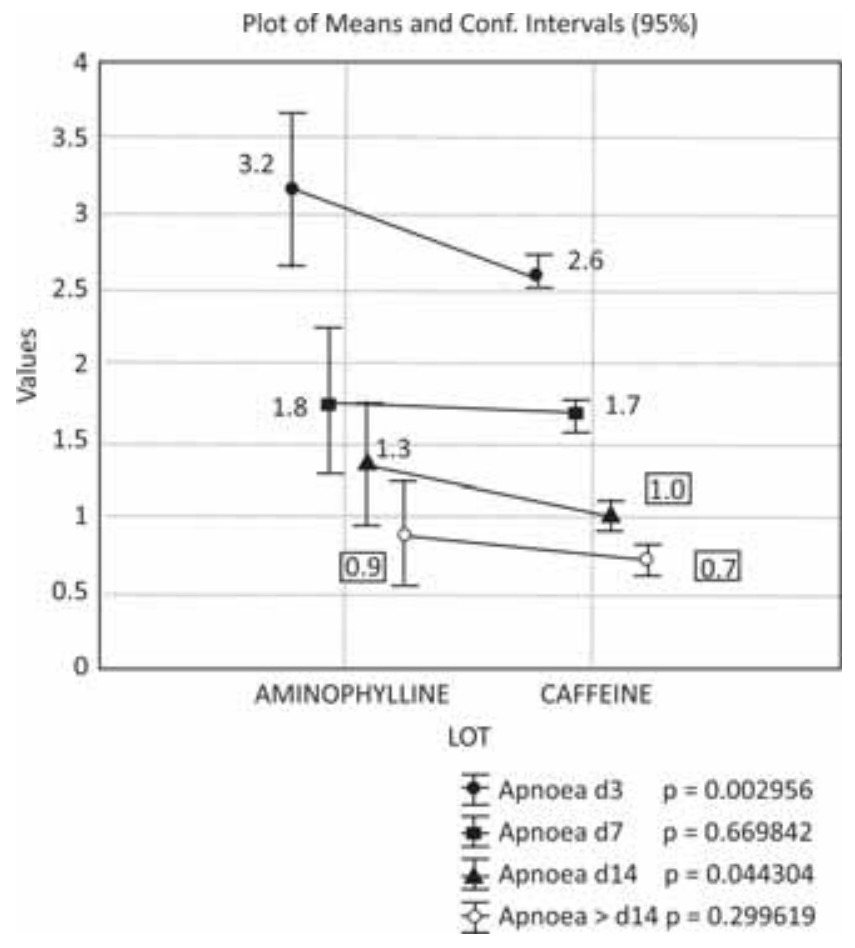

FIGURE 2. Frequency of apnoeic events after the initiation of methylxanthine treatment

The mean duration of aminophylline treatment was 15.98 days, whereas for caffeine, 22.8 days $(\mathrm{p}=0.006454 ; \mathrm{CI}=95 \%)$.

Concerning the severity of apnoea, in both groups the events that required intervention (tactile stimulation, positive pressure ventilation or CPAP) were predominant $-86.96 \%$ in group 1 vs. $88.37 \%$ in group 2 - while the events with spontaneous resolution had a prevalence of $13.04 \%$ and $11.63 \%$, respectively. As for the required measures, in the caffeine group tactile stimulation was reported in $91.36 \%$ of the events vs. $60.87 \%$ in the aminophylline group $(p<<0.001 ; C I=95 \%)$. In return, the neonates from the aminophylline group required PPV and CPAP more frequently (5.32\% vs. $2.17 \%$; 


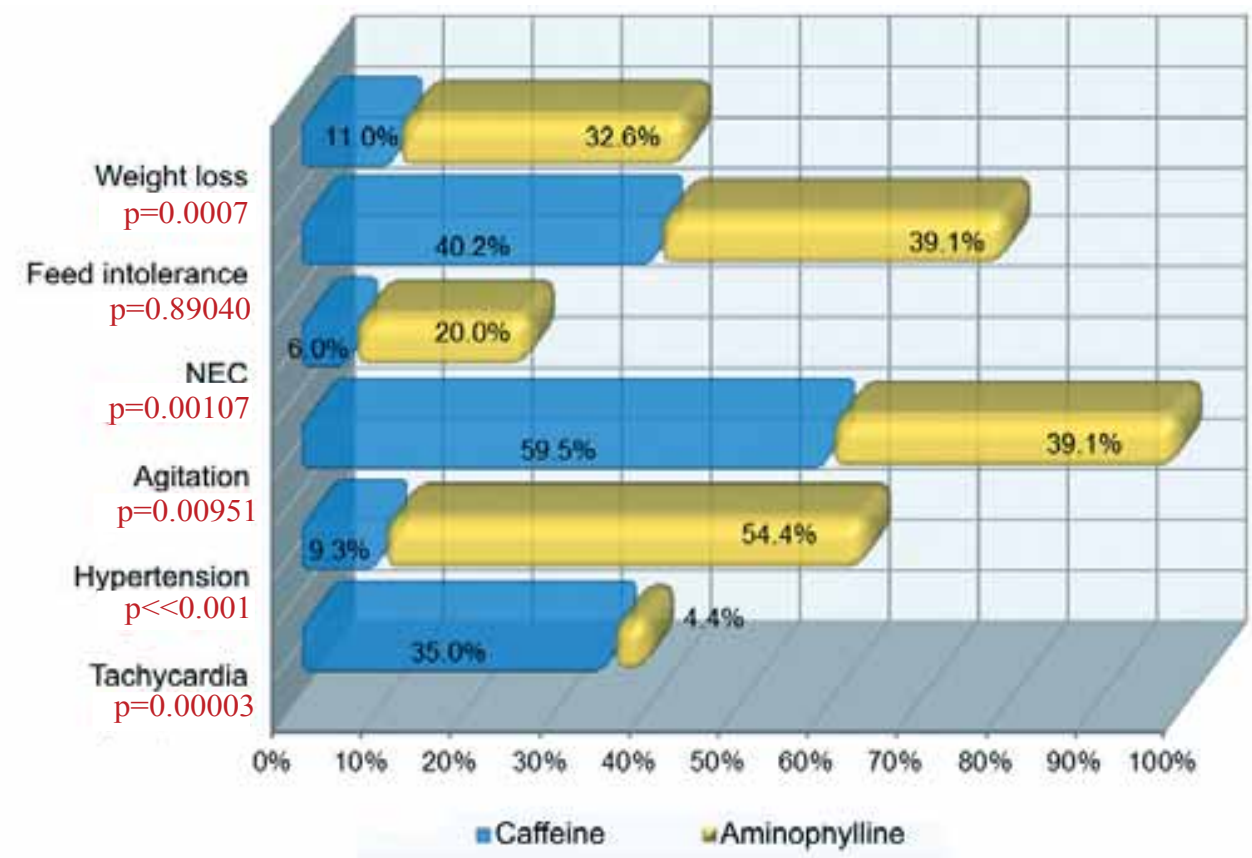

FIGURE 3. Comparative analysis of the methylxanthine treatment side effects $N E C$ - necrotizing enterocolitis

$\mathrm{p}=0.35788 ; \mathrm{CI}=95 \%$ and $63.04 \%$ vs. $49.50 \%$; $\mathrm{p}=0.08701 ; \mathrm{CI}=95 \%$, respectively).

We also analysed the incidence of the side effects of the methylxantine treatment comparatively in both groups, and found a higher incidence of agitation, tachycardia and digestive intolerance in premature infants treated with caffeine, but a lower incidence of NEC (necrotizing enterocolitis), weight loss and hypertension compared to those in the aminophylline group (Fig. 3).
Knowing the fact that anaemia, intraventricular haemorrhage, patent ductus arteriosus and chronic lung disease are involved in the pathogenesis of apnoea, we analysed their incidence in both study groups (Fig. 4) and also their influence of the duration of methylxanthine treatment (Fig. 5).

The mean duration for hospital admission of the infants in the aminophylline group was 69.85 days vs. 59.33 days in the caffeine group $(\mathrm{p}=0.0052$; $\mathrm{CI}=95 \%)$.

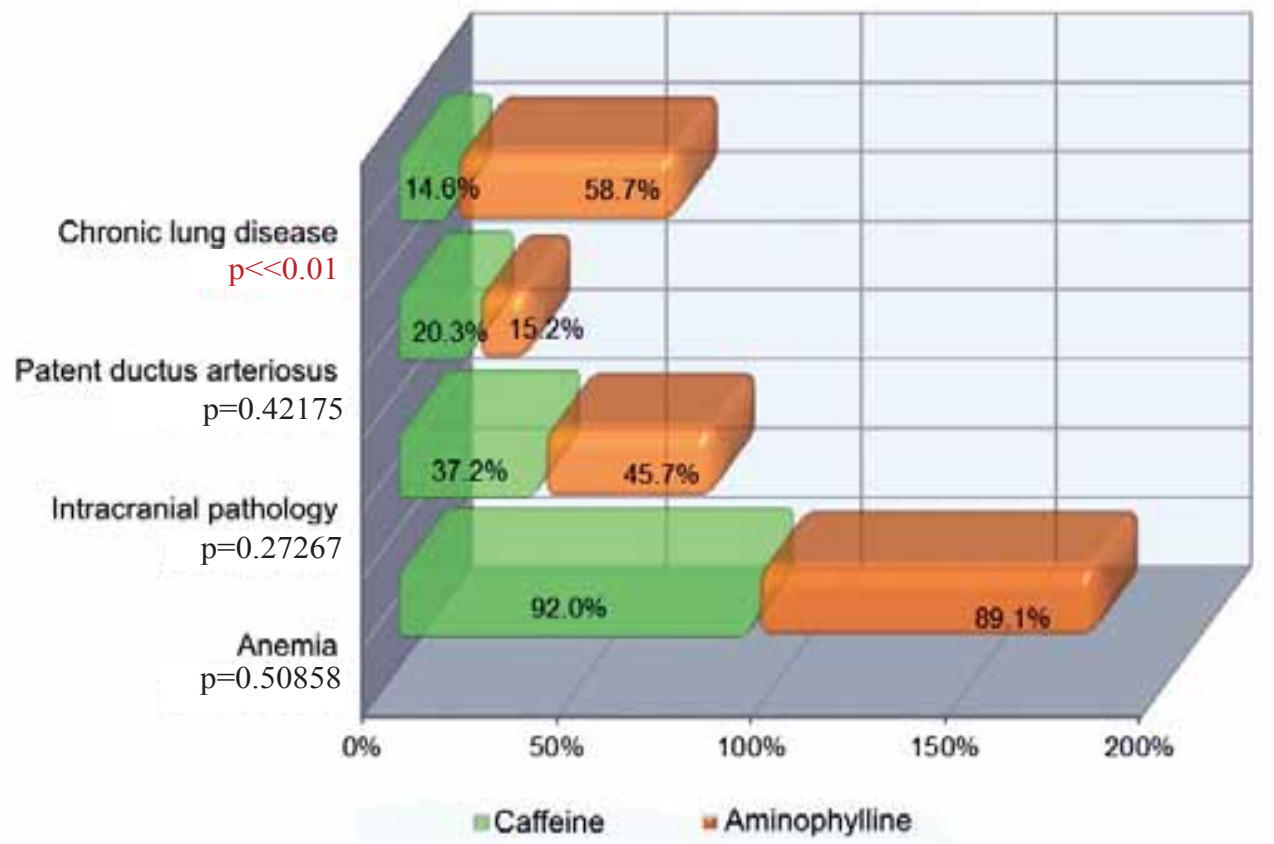

FIGURE 4. Associated pathology (comparison between study groups) 
Categ. Box \& Whisker Plot: Treatment duration (days) Treatment duration (days): $F(1.345)=8.8078, p=0.0032$; $\mathrm{KW}-\mathrm{H}(1.347)=11.0483, \mathrm{p}=0.0009$

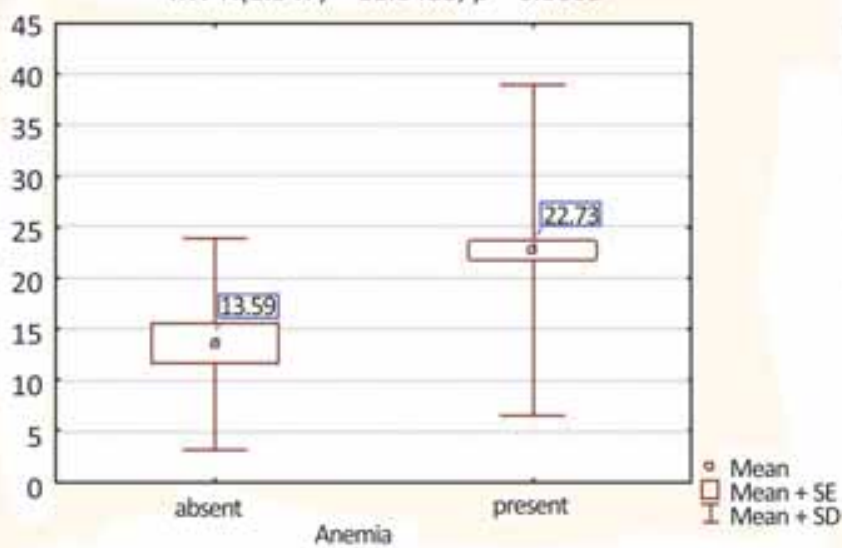

Categ. Box \& Whisker Plot: Treatment duration (days) Treatment duration (days): $F(1.345)=15.5675, p=0.00010$;

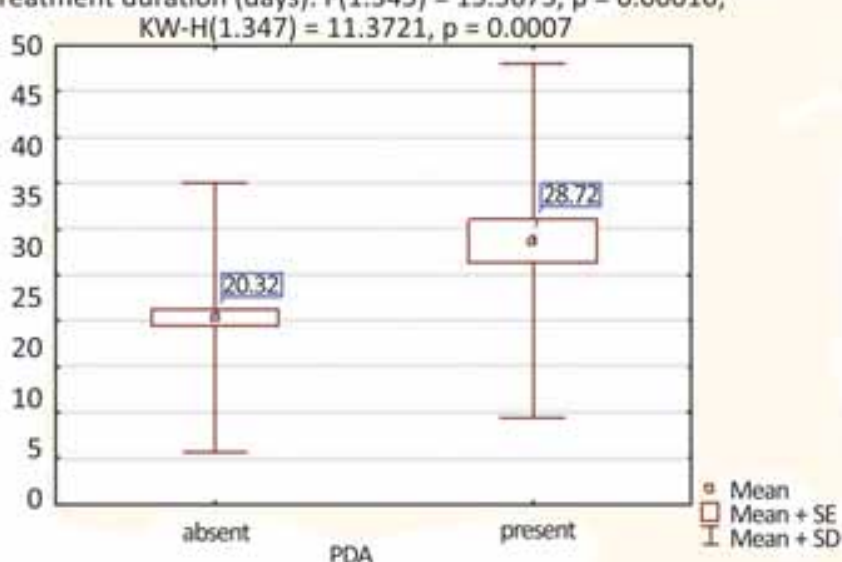

Categ. Box \& Whisker Plot: Treatment duration (days) Treatment duration (days): $F(1.345)=10.737, p=0.0012$; $\mathrm{KW}-\mathrm{H}(1.347)=11.7082, \mathrm{p}=0.0006$

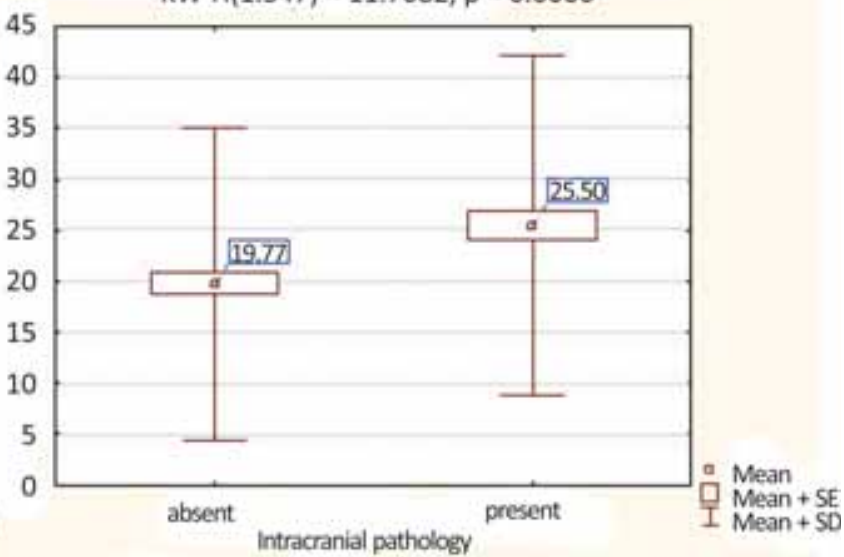

Categ. Box \& Whisker Plot: Treatment duration (days) Treatment duration (days): $F(1.345)=15.6201, p=0.0008$;

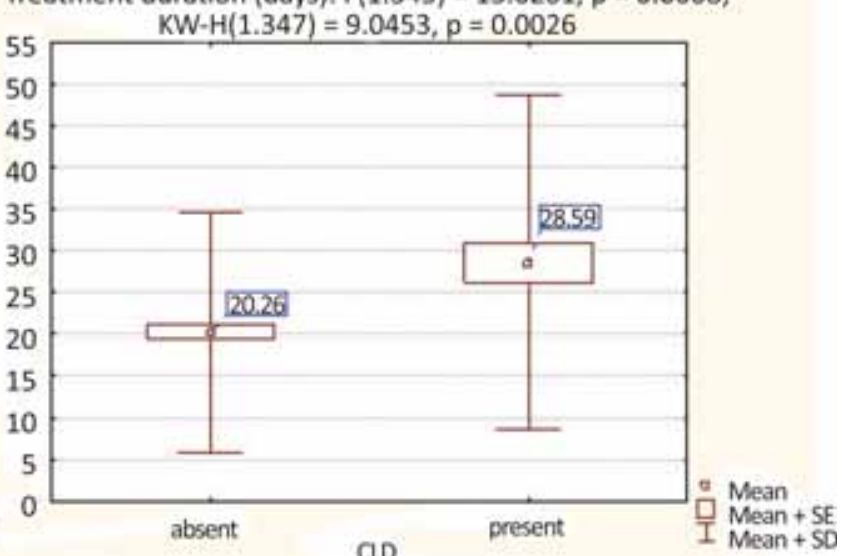

FIGURE 5. Correlation between associated pathology and treatment duration

$P D A$ - patent ductus arteriosus

CLD - chronic lung disease

\section{DISCUSSION}

Approximately $70 \%$ of babies born before 34 weeks of gestation have clinically significant apnoea, bradycardia, or desaturation during their hospital stay. Apnoea may occur during the postnatal period in $25 \%$ of neonates who weighed less than $2,500 \mathrm{~g}$ at birth and in $84 \%$ of neonates who weigh less than $1,000 \mathrm{~g} \mathrm{(10).} \mathrm{Carlo} \mathrm{and} \mathrm{Barrington} \mathrm{showed}$ that apnoea may begin on the first day of life in neonates without respiratory distress syndrome $(11,12)$. The incidence of apnoea varies depending on the gestational age. Martin et al. reported that $7 \%$ of neonates born at 34 to 35 weeks gestation, $15 \%$ at 32 to 33 weeks, $54 \%$ at 30 to 31 weeks (13), while Robertson et al. established that nearly all infants born at $<29$ weeks gestation or $<1,000 \mathrm{~g}$ exhibit AOP (14). However, it's important to keep in mind that apnoea of prematurity is always a diagnosis of exclusion, as many conditions like intrapartum magnesium exposure, systemic infections or the fetal inflammatory response syndrome, pne- umonia, intracranial pathology, seizures, hypoglycemia and other metabolic disturbances may manifest with apnoea (10). The incidence of apnoea reported by our study, respectively $61.38 \%(\leq 29$ wks.), 43.03\% (30-31 wks.), 18.8\% (32-33 wks.) and $2.99 \%$ (34-35 wks.), are lower comparing with those stated in the studies mentioned above.

Of all neonates admitted in our unit between June 2013 and June 2015, we included in the study premature infants with $\mathrm{GA}<35$ weeks who required methylxanthines for prophylaxis or treatment of apnoea or to facilitate extubation.

The administration protocols for the two methylxanthines were different. In the aminophylline group, the loading dose was administered at extubation, followed by the maintenance dose until the apnoea resolved. In the caffeine group, depending on the gestational age, the pathology and clinical status of the neonate, the loading dose was given either in the first 3 days of life, at extubation or as treatment for apnoea, followed by a daily maintenance dose until 34-36 weeks postconceptional age 
or until the apnoea resolved, resulting in a longer administration of caffeine, compared to aminophylline.

Neonates that received caffeine had a significant reduction of apnoea on day 3 and 14 of treatment compared to those in the aminophylline group, with no statistically relevant difference on day 7 and after the $14^{\text {th }}$ day of treatment. This proves the efficiency of methylxanthines for AOP treatment and a quicker effect of caffeine compared to aminophylline. Overall, on the short-term, caffeine and aminophylline seem to have similar effects in reducing apnoea/bradycardia, as reported by other authors (8).

Weaning and extubating preterm infants on mechanical ventilation may be difficult because of the relatively poor respiratory drive and tendency to develop hypercarbia and apnoea, particularly in very preterm infants. Thus, methylxanthine treatment started before extubation may be effective in facilitating removal of the respiratory support in ventilated neonates by stimulating breathing and reducing postextubation apnoea (15). Our study also showed that caffeine treatment was associated with a slight decrease of need for CPAP and mechanical ventilation compared to aminophylline, but the differences were not statistically relevant.

On the long-term, our study showed that in the caffeine group the incidence of chronic lung disease was significantly lower compared to the aminophylline group, in accordance with the data reported by other authors (15). On the other hand, anaemia, intraventricular haemorrhage, persistent ductus arteriosus and chronic lung disease were statistically correlated with a prolonged duration of treatment for apnoea of prematurity.

Analysis of the side effects of mehylxanthines showed that neonates from the caffeine group had more episodes of agitation, tachycardia and feed intolerance, but aminophylline treatment was associated with a higher incidence of NEC, hypertension and weight loss. Henderson-Smart and Steer (8) performed a meta-analysis of 5 studies that in- cluded 108 premature infants with a mean GA of 30 weeks, who received methylxanthines for AOP (54 - caffeine, 54 - teophylline). The authors concluded that theophylline was associated with a higher toxicity rate (tachycardia, agitation, feed intolerance, NEC, poor weight gain). Our result was not concurrent with the existing reports, but this could be explained by the much smaller number of infants in the aminophylline group (46), compared to those in the caffeine group (301).

The shorter hospital stay of infants from the caffeine group compared to those in aminophylline group in addition to the reduction in the need for invasive and non-invasive respiratory support, pleads for a better cost/benefit ratio in the case of caffeine, which recommends it as the drug of choice for treatment of apnoea in premature infants.

\section{CONCLUSION}

Both caffeine and aminophylline have proved to be efficient in reducing the number of apnoea and facilitation of weaning of the ventilator and extubation of extremely premature infants. Also, caffeine administration was correlated with lower rates of bronchopulmonary dysplasia.

The therapeutic advantages of caffeine compared to aminophylline, including better enteral absorption, longer half-life which makes it efficient in a single daily dose, lower rate of side effects and a favourable long-term cost/benefit ratio, recommend it as the first choice drug for the treatment of apnoea in premature neonates.

Considering the advantages of caffeine treatment against the higher cost comparing to aminophylline, it would be extremely useful to develop a set of national guidelines regarding prophylaxis and treatment for apnoea in premature infants, as well as a broader multicentre study on a larger number of neonates, in order to establish the efficiency and safety of caffeine administration on the long term neurodevelopment and growth.

\section{REFERENCES}

1. Committee on Fetus and Newborn. American Academy of Pediatrics. Apnea, sudden infant death syndrome, and home monitoring. Pediatrics 2003; 111: 914-917.

2. Arora P. Pathogenesis and Management of Apnea of Prematurity: A Brief Overview. J Neonatal Bio 2012; 1:e104.

3. Mueni E., Opiyo N., English M. Caffeine for the management of apnea in preterm infants Int Health 2009; 1(2):190-195.

4. Atkinson E., Fenton A.C. Management of apnoea and bradycardia in neonates Paediatrics and Child Health 2009; 19(12):550-554.

5. Zhao J., Gonzalez F., Mu D. Apnea of prematurity: from cause to treatment Eur J Pediatr 2011; 170:1097-1105.

6. Henderson-Smart D.J., De Paoli A.G. Methylxanthine treatment for apnoea in preterm infants. Cochrane Database Syst Rev 2010; Issue 12. Art. No.: CD000140. 
7. Henderson-Smart D.J. Recurrent apnoea. Evidence Based Pediatrics. Oxford: Blackwell, 2004.

8. Henderson-Smart D.J., Steer P.A. Caffeine versus theophylline for apnea in preterm infants. Cochrane Database Syst Rev 2010; Issue 1. Art. No.: CD000273.

9. Jobe A.H., Bancalari E. Bronchopulmonary displasia. Am J Respir care Med, 2001; 163(7):1723-9.

10. Nimavat D.J., Sherman M.P., Santin R.L. Apnea of Prematurity. http://emedicine.medscape.com/article/97497, updated May 29, 2014, accessed November 20, 2015.

11. Carlo W.A., Martin R.J., Versteegh F.G., et al. The effect of respiratory distress syndrome on chest wall movements and respiratory pauses in preterm infants. Am Rev Respir Dis. $1982 \mathrm{Jul}$. 126(1):103-7.
12. Barrington K.J., Finer N.N. Periodic breathing and apnea in preterm infants. Pediatr Res. 1990 Feb. 27(2):118-21.

13. Martin R.J., Abu Shaweesh J.M., Baird T.M. Apnoea of prematurity. Paediatr Respir Rev. 2004; 5(Suppl 1):S377-S382.

14. Robertson C.M., Watt M.J., Dinu I.A. Outcomes for the extremely premature infant: what is new? And where are we going? Pediatr Neurol. 2009; 40(3):189-196.

15. Henderson-Smart D.J., Davis P.G. Prophylactic methylxanthines for endotracheal extubation in preterm infants. Cochrane Database Syst Rev 2010; Issue 12. Art. No.: CD000139. 